\title{
Rational Hopf $G$-spaces with two nontrivial homotopy group systems
}

\author{
by
}

\author{
Ryszard Doma n (Poznań)
}

\begin{abstract}
Let $G$ be a finite group. We prove that every rational $G$-connected Hopf $G$-space with two nontrivial homotopy group systems is $G$-homotopy equivalent to an infinite loop $G$-space.
\end{abstract}

1. Introduction. It is known that a rational $H$-space $X$ of the homotopy type of a connected $C W$-complex is homotopy equivalent to a weak product of Eilenberg-MacLane spaces [3], and thus to an infinite loop space. In this paper we study the question of whether there is an analogous result for $X$ admitting a finite group action compatible with the $H$-structure.

Let $G$ be a finite group. $G$-spaces, $G$-maps and $G$-homotopies considered in this paper will be pointed. We shall work in the category of $G$-spaces having the $G$-homotopy type of $G$-CW-complexes [1]. In case of need, we shall tacitly replace $G$-spaces by their $G-C W$-substitutes. We shall also assume all $G$-spaces to be $G$-connected in the sense that all the fixed point spaces $X^{H}$ are connected for all subgroups $H$ of $G$.

Definition. A Hopf $G$-space is a Hopf space $X$ on which $G$ acts in such a way that the multiplication $m: X \times X \rightarrow X$ is $G$-equivariant, and the composite $X \vee X \subset X \times X \stackrel{m}{\rightarrow} X$ is $G$-homotopic to the folding map.

For example, if $Y$ is a $G$-space, then the loop space $\Omega Y$ is a Hopf $G$-space, where the action of $G$ is defined by $(g f)(t)=g(f(t))$.

Let $X$ be a $G$-simple $G$-space (i.e. each $X^{H}$ is simple). We shall call $X$ rational if the homotopy groups $\pi_{i}\left(X^{H}\right)$ are $\mathbb{Q}$-vector spaces for each subgroup $H$ of $G$. Note that, by [4], every $G$-simple $G$-space, in particular Hopf $G$-space, can be rationalized. Moreover, in the latter case, the resulting $G$-space is a Hopf $G$-space.

1991 Mathematics Subject Classification: Primary 55P45, 55P62, 55P91.

Supported by Polish scientific grant RP.I.10. 
Let $O_{G}$ be the category of canonical orbits of $G$ and $G$-maps between them. A coefficient system for $G$ is a contravariant functor from $O_{G}$ to the category of abelian groups. We shall call a coefficient system rational if its range is the category of $\mathbb{Q}$-vector spaces. For a $G$-space $X$, the homotopy and homology group systems $\underline{\pi}_{n}(X)$ and $\underline{\widetilde{H}}_{n}(X)$ are defined by

$$
\underline{\pi}_{n}(X)(G / H)=\pi_{n}\left(X^{H}\right), \quad \underline{\widetilde{H}}_{n}(X)(G / H)=\widetilde{H}_{n}\left(X^{H}\right),
$$

where $\widetilde{H}_{n}$ denotes the reduced singular homology with $\mathbb{Z}$-coefficients.

Given a coefficient system $M$ for $G$ and an integer $m \geq 1$, an EilenbergMacLane $G$-space of type $(M, n)$ is a $G$-space $X$ such that $\underline{\pi}_{m}(X)=M$ and $\underline{\pi}_{q}(X)=0$ for $q \neq m$. Such $G$-spaces always exist and are unique up to $G$-homotopy equivalence [1].

We shall call a $G$-space $X^{0}$ an infinite loop $G$-space if there exist a sequence $X^{0}, X^{1}, X^{2}, \ldots$ of $G$-spaces and a sequence $f_{n}: X^{n} \rightarrow \Omega X^{n+1}$, $n \geq 0$, of $G$-homotopy equivalences.

Eilenberg-MacLane $G$-spaces ere examples of infinite loop $G$-spaces. Moreover, using the $G$-obstruction argument [1], it can be shown that a $G$-Hopf structure on an Eilenberg-MacLane $G$-space is unique up to $G$ homotopy. We shall denote an Eilenberg-MacLane $G$-space of type $(M, m)$ by $K(M, m)$. Recall also that Eilenberg-MacLane $G$-spaces represent Bredon cohomology [1]: $\widetilde{H}_{G}^{m}(X, M)=[X, K(M, m)]_{G}$, where [, $]_{G}$ denotes the $G$-homotopy classes of $G$-maps. The relation between $\widetilde{H}_{G}^{m}(X, M)$ and $\underline{\widetilde{H}}_{*}(X)$ is given by a spectral sequence with $E_{2}^{p, q}=\operatorname{Ext}^{p}\left(\underline{\widetilde{H}}_{q}(X), M\right) \Rightarrow \widetilde{H}_{G}^{p+q}(X, M)$ [1]. We shall refer to it as the Bredon spectral sequence.

Let $\mathbb{Z} / p^{k}$ denote the cyclic group of order $p^{k}$, where $p$ is prime and $k$ is a positive integer. A theorem by G. Triantafillou [5] states that each rational $\mathbb{Z} / p^{k}$-connected Hopf $\mathbb{Z} / p^{k}$-space is $\mathbb{Z} / p^{k}$-homotopy equivalent to a weak product of Eilenberg-MacLane $\mathbb{Z} / p^{k}$-spaces, and hence to an infinite loop $\mathbb{Z} / p^{k}$-space. In contrast to the nonequivariant case, however, rational Hopf $G$-spaces do not split equivariantly into a product of Eilenberg-MacLane $G$-spaces in general [5]. Nevertheless, the counterexamples given in [5] are still infinite loop $G$-spaces. This gives rise to the question of whether every rational Hopf $G$-space $X$ is $G$-homotopy equivalent to an infinite loop $G$ space.

In the present paper we answer the above question affirmatively in the case where $X$ has only two nontrivial homotopy group systems. Thus we prove the following

Theorem. Let $X$ be a rational $G$-connected Hopf $G$-space having only two nontrivial homotopy group systems. Then $X$ is $G$-homotopy equivalent to an infinite loop $G$-space. 
2. Equivariant $k$-invariants of Hopf $G$-spaces. Let $X$ be a Hopf $G$-space with a $G$-multiplication $m: X \times X \rightarrow X$ and let $N$ be a coefficient system for $G$. An element $u$ of $\widetilde{H}_{G}^{n}(X, N)$ is called primitive if $m^{*}(u)=$ $p_{1}^{*}(u)+p_{2}^{*}(u)$ in $\widetilde{H}_{G}^{n}(X \times X, N)$, where $p_{1}$ and $p_{2}$ are the two projections.

Now, let

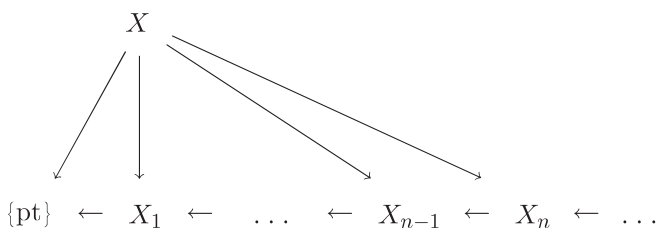

be an equivariant Postnikov decomposition of $X$ (see [4]). We shall use the following results of [5]:

Proposition 2.1. Each $X_{n}$ is a Hopf G-space.

Proposition 2.2. The equivariant $k$-invariant $k^{n+1} \in \widetilde{H}_{G}^{n}\left(X_{n-1}, \underline{\pi}_{n}(X)\right)$ is primitive for all $n \geq 1$.

3. Primitive elements in the Bredon cohomology of rational Eilenberg-MacLane $G$-spaces. For a Hopf $G$-space $Y$ with a $G$-multiplication $\mu: Y \times Y \rightarrow Y$ and a coefficient system $N$ for $G$, consider the homomorphism $t^{*}=\mu^{*}-p_{1}^{*}-p_{2}^{*}: \widetilde{H}_{G}^{n}(Y, N) \rightarrow \widetilde{H}_{G}^{n}(Y \times Y, N)$, where $p_{1}$ and $p_{2}$ are the two projections. Let $\widetilde{H}_{G}^{n}(Y, N)=J^{0, n} \supset \ldots \supset J^{n, 0}=0$ and $\widetilde{H}_{G}^{n}(Y \times Y, N)=F^{0, n} \supset \ldots \supset F^{n, 0}=0$ be the filtrations corresponding to the Bredon spectral sequences converging to $\widetilde{H}_{G}^{n}(Y, N)$ and $\widetilde{H}_{G}^{n}(Y \times Y, N)$, respectively. By our assumptions about $G$-spaces, the $G$ cellular approximation theorem [1], and the construction of the Bredon spectral sequence, the homomorphism $t^{*}$ preserves the filtrations, and the induced homomorphism $E_{\infty}^{p, q}(Y) \rightarrow E_{\infty}^{p, q}(Y \times Y)$ can be identified with the one induced by $\operatorname{Ext}^{p}\left(t_{*}, N\right): \operatorname{Ext}^{p}\left(\widetilde{\widetilde{H}}_{q}(Y), N\right) \rightarrow \operatorname{Ext}^{p}\left(\widetilde{\widetilde{H}}_{q}(Y \times Y), N\right)$, where $t_{*}=\mu_{*}-p_{1 *}-p_{2 *}: \underline{\widetilde{H}}_{q}(Y \times Y) \rightarrow \underline{\widetilde{H}}_{q}(Y)$ is a morphism of coefficient systems. We can summarize the above in

Proposition 3.1. The homomorphism $t^{*}: \widetilde{H}_{G}^{n}(Y, N) \rightarrow \widetilde{H}_{G}^{n}(Y \times Y, N)$ is the limit of a morphism of the Bredon spectral sequences whose $E_{2}^{p, q}$-term is $\operatorname{Ext}^{p}\left(t_{*}, N\right): \operatorname{Ext}^{p}\left(\underline{\tilde{H}}_{q}(Y), N\right) \rightarrow \operatorname{Ext}^{p}\left(\underline{\widetilde{H}}_{q}(Y \times Y), N\right)$. We are now going to examine the morphism $t_{*}: \underline{\widetilde{H}}_{q}(Y \times Y) \rightarrow \underline{\widetilde{H}}_{q}(Y)$ for $Y$ being a rational Eilenberg-MacLane $G$-space.

Proposition 3.2. Let $Y$ be an Eilenberg-MacLane G-space of type $(M, m)$. Then the morphism $t_{*}: \underline{\widetilde{H}}_{q}(Y \times Y) \rightarrow \underline{\widetilde{H}}_{q}(Y)$ has a right inverse for each $q \neq m$. 
Pr o o f. For each subgroup $H$ of $G$, the fixed point space $Y^{H}$ is a rational Eilenberg-MacLane $G$-space of type $(M(G / H), m)$. Thus, by [2, Appendix], the Pontryagin algebra $H_{*}\left(Y^{H}\right)$ is the free graded commutative algebra generated by $\widetilde{H}_{m}\left(Y^{H}\right)$. In particular, the multiplication $\mu_{*}^{H}: H_{*}\left(Y^{H}\right) \otimes$ $H_{*}\left(Y^{H}\right) \rightarrow H_{*}\left(Y^{H}\right)$ is a graded algebra homomorphism. Now suppose that $a_{1}, \ldots, a_{k}$ belong to $\widetilde{H}_{m}\left(Y^{H}\right)$, and let $a_{1} \ldots a_{k} \in \widetilde{H}_{k m}\left(Y^{H}\right)$ be their product. Let $\Delta^{H}: Y^{H} \rightarrow Y^{H} \times Y^{H}$ be the diagonal map. Since every element of $H_{m}\left(Y^{H}\right)$ is primitive, we have

$$
\begin{aligned}
\left(\mu_{*}^{H}-p_{1 *}^{H}-\right. & \left.p_{2 *}^{H}\right) \Delta_{*}^{H}\left(a_{1} \ldots a_{k}\right)=\mu_{*}^{H} \Delta_{*}^{H}\left(a_{1} \ldots a_{k}\right)-2 a_{1} \ldots a_{k} \\
& =\mu_{*}^{H}\left(\left(a_{1} \otimes 1+1 \otimes a_{1}\right) \ldots\left(a_{k} \otimes 1+1 \otimes a_{k}\right)\right)-2 a_{1} \ldots a_{k} \\
& =\left(2^{k}-2\right) a_{1} \ldots a_{k} .
\end{aligned}
$$

This implies that $\left(1 /\left(2^{k}-2\right)\right) \Delta_{*}: \underline{\widetilde{H}}_{k m}(Y) \rightarrow \underline{\widetilde{H}}_{k m}(Y \times Y)$, where $\Delta:$ $Y \rightarrow Y \times Y$ is the diagonal, is a right inverse of $t_{*}$ for each $k \neq 1$. Since $\underline{\widetilde{H}}_{q}(Y)=\underline{\widetilde{H}}_{q}(Y \times Y)=0$ for $q \neq k m$, the desired result follows.

For each element $u \in \widetilde{H}_{G}^{n}(Y, N)$, define the weight $w(u)$ of $u$ to be the greatest lower bound of the integers $q$ such that $u \in J^{n-q, q}$, where $\widetilde{H}_{G}^{n}(Y, N)=J^{0, n} \supset \ldots \supset J^{n, 0}=0$ is the filtration corresponding to the Bredon spectral sequence.

Proposition 3.3. Suppose that $Y$ is an Eilenberg-MacLane $G$-space of type $(M, m)$, where $M$ is a rational coefficient system for $G$. Then $w(u) \leq m$ for every primitive element $u$ of $\widetilde{H}_{G}^{n}(Y, N)$.

Proof. Let $\left\{J^{p, n-p}(Y)\right\}$ and $\left\{J^{p, n-p}(Y \times Y)\right\}$ be the filtrations of $\widetilde{H}_{G}^{n}(Y, N)$ and $\widetilde{H}_{G}^{n}(Y \times Y)$, respectively, which correspond to the Bredon spectral sequences. Suppose that $u \in \widetilde{H}_{G}^{n}(Y, N)$ is primitive and set $w(u)=$ $q$. Consider the commutative diagram

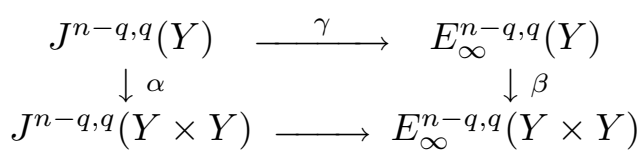

where $\alpha$ is the restriction of $t^{*}: \widetilde{H}_{G}^{n}(Y, N) \rightarrow \widetilde{H}_{G}^{n}(Y \times Y), \beta$ is induced by $\operatorname{Ext}^{n-q}\left(t_{*}, N\right): \operatorname{Ext}^{n-q}\left(\underline{\widetilde{H}}_{q}(Y), N\right) \rightarrow \operatorname{Ext}^{n-q}\left(\underline{\widetilde{H}}_{q}(Y \times Y), N\right)$, and $\gamma$ is the projection. If $w(u)>m$ then, by Proposition 3.2, $\beta$ is a monomorphism. Thus $\beta \gamma(u) \neq 0$. Consequently, $u$ cannot be primitive.

4. Proof of Theorem. Let $X$ be a rational Hopf $G$-space having only two nontrivial homotopy group systems $\underline{\pi}_{m}(X)=M$ and $\underline{\pi}_{n}(X)=$ $N, m<n$. Then $X$ is determined by its equivariant $k$-invariant $k(X) \in$ 
$\widetilde{H}_{G}^{n+1}(K(M, m), N)$, which, by Proposition 2.2 , is primitive. The cohomology suspension $\sigma^{*}: \widetilde{H}_{G}^{q+1}(K(M, r+1), N) \rightarrow \widetilde{H}_{G}^{q}(K(M, r), N)$, which corresponds to the map $\Omega:[K(M, r+1), K(N, q+1)]_{G} \rightarrow[K(M, r), K(N, q)]_{G}$, is, by Lemma 3.3 of [5], the limit of a morphism of spectral sequences with $E_{2}$-term

$$
\operatorname{Ext}^{i}\left(\sigma_{*}, N\right): \operatorname{Ext}^{i}\left(\underline{\widetilde{H}}_{j+1}(K(M, r+1)), N\right) \rightarrow \operatorname{Ext}^{i}\left(\underline{\widetilde{H}}_{j}(K(M, r)), N\right),
$$

where $\sigma_{*}: \underline{\widetilde{H}}_{j}(K(M, r)) \rightarrow \underline{\widetilde{H}}_{j+1}(K(M, r+1))$ is determined by homology suspension.

In order to prove the Theorem we only need to show that the equivariant $k$-invariant $k(X)$ belongs to the image of the composite

$$
\begin{aligned}
\widetilde{H}_{G}^{n+k}(K(M, m+k-1), N) \rightarrow \widetilde{H}_{G}^{n+k-1} & (K(M, m+k-2), N) \\
& \rightarrow \ldots \rightarrow \widetilde{H}_{G}^{n+1}(K(M, m), N)
\end{aligned}
$$

of cohomology suspensions for each $k>1$.

By Proposition 3.3, we know that $w(k(X)) \leq m$. Thus the proof of the Theorem will be completed if we prove the following

Proposition 4.1. Let $\widetilde{H}_{G}^{q+1}(K(M, r+1), N)=F^{0, q+1} \supset \ldots \supset F^{q+1,0}=$ 0 and $\widetilde{H}_{G}^{q}(K(M, r), N)=J^{0, q} \supset \ldots \supset J^{q+1,0}=0$ be the filtrations corresponding to the Bredon spectral sequences, where $q \geq n+1$ and $r=m+q-n$ -1 . Then the cohomology suspension $\sigma^{*}: \widetilde{H}_{G}^{q+1}(K(M, r+1), N) \rightarrow$ $\widetilde{H}_{G}^{q}(K(M, r), N)$ restricted to $F^{q-r, r+1}$ gives an isomorphism $\widetilde{\sigma}^{*}: F^{q-r, r+1}$ $\rightarrow J^{q-r, r}$.

Proof. Denote by $E_{*}^{*, *}$ the Bredon spectral sequence converging to $\widetilde{H}_{G}^{q+1}(K(M, r), N)$, and by ' $E_{*}^{*, *}$ the one converging to $\widetilde{H}_{G}^{q}(K(M, r), N)$. We have

$$
E_{2}^{q-1, r+1}=\operatorname{Ext}^{q-1}\left(\underline{\tilde{H}}_{r+1}(K(M, r+1)), N\right)
$$

and

$$
{ }^{\prime} E_{2}^{q-r, r}=\operatorname{Ext}^{q-r}\left(\underline{\widetilde{H}}_{r+1}(K(M, r)), N\right) .
$$

Hence $E_{\infty}^{q-r, r+1} \cong F^{q-r, r+1}$ and ' $E_{\infty}^{q-r, r} \cong J^{q-r, r}$. Under the above identification, $\widetilde{\sigma}^{*}$ is induced by $\sigma_{*}: \underline{\widetilde{H}}_{r}(K(M, r)) \rightarrow \underline{\widetilde{H}}_{r+1}(K(M, r+1))$. Since, evidently, $\sigma_{*}$ is an isomorphism, so is $\widetilde{\sigma}^{*}$.

Re m ark 4.2. Since we have not used the assumption that the coefficient system $N$ is rational, the conclusion of the Theorem is valid for a Hopf $G$ space $X$ having only two nontrivial homotopy group systems $\underline{\pi}_{m}(X)$ and $\underline{\pi}_{n}(X), m<n$, with $\underline{\pi}_{m}(X)$ rational. 


\section{References}

[1] G. E. Bredon, Equivariant Cohomology Theories, Lecture Notes in Math. 34, Springer, 1967.

[2] J. W. Milnor and J. C. Moore, On the structure of Hopf algebras, Ann. of Math. 81 (1965), 211-264.

[3] H. Scheerer, On rationalized $H$ - and co-H-spaces, Manuscripta Math. 51 (1984), 63-87.

[4] G. W. Triantafillou, Equivariant minimal models, Trans. Amer. Math. Soc. 274 (1982), 509-532.

[5] -, Rationalization of Hopf G-spaces, Math. Z. 182 (1983), 485-500.

FACULTY OF MATHEMATICS AND COMPUTER SCIENCE

ADAM MICKIEWICZ UNIVERSITY

MATEJKI 48/49

60-769 POZNAŃ, POLAND

Received 2 September 1992;

in revised form 15 March 1994 